\title{
Determining internal screening electric field of working polymer light emitting diodes
}

Katsuichi Kanemoto, Takahiro Takahashi, Hideki Hashimoto

\begin{tabular}{|c|l|}
\hline Citation & Applied Physics Letters, 109(1);013301 \\
\hline Issue Date & 2016-07-05 \\
\hline Type & Journal Article \\
\hline Textversion & publisher \\
\hline \multirow{3}{*}{ Rights } & $\begin{array}{l}\text { C } 2016 \text { Author(s). Published by AIP Publishing. } \\
\text { This article may be downloaded for personal use only. Any other use requires prior } \\
\text { permission of the author and AIP Publishing. The following article appeared in } \\
\text { Applied Physics Letters and maybe found at https://doi.org/10.1063/1.4955126 }\end{array}$ \\
\hline DOI & $10.1063 / 1.4955126$ \\
\hline
\end{tabular}

Self-Archiving by Author(s)

Placed on: Osaka City University

Katsuichi Kanemoto, Takahiro Takahashi, Hideki Hashimoto. (2016). Determining internal screening electric field of working polymer light emitting diodes. Applied Physics Letters. 109. 013301. 


\section{Determining internal screening electric field of working polymer light emitting diodes}

Cite as: Appl. Phys. Lett. 109, 013301 (2016); https://doi.org/10.1063/1.4955126

Submitted: 29 January 2016 . Accepted: 20 June 2016 . Published Online: 05 July 2016

Katsuichi Kanemoto, Takahiro Takahashi, and Hideki Hashimoto

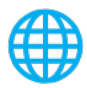

\section{ARTICLES YOU MAY BE INTERESTED IN}

Organic electroluminescent diodes

Applied Physics Letters 51, 913 (1987); https://doi.org/10.1063/1.98799

Direct measurement of electric field screening in light emitting diodes with conjugated polyelectrolyte electron injecting/transport layers

Applied Physics Letters 94, 033301 (2009); https://doi.org/10.1063/1.3069279

Solution-processed multilayer polymer light-emitting diode without intermixing

Applied Physics Letters 110, 023302 (2017); https://doi.org/10.1063/1.4973989
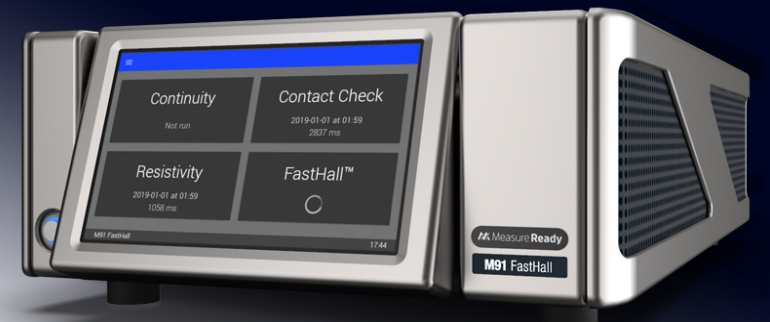

\section{Measure Ready} M91 FastHall ${ }^{\mathrm{TM}}$ Controller

A revolutionary new instrument for complete Hall analysis 


\title{
Determining internal screening electric field of working polymer light emitting diodes
}

\author{
Katsuichi Kanemoto, ${ }^{1, a)}$ Takahiro Takahashi, ${ }^{1}$ and Hideki Hashimoto ${ }^{1,2, b)}$ \\ ${ }^{1}$ Department of Physics, Graduate School of Science, Osaka City University, 3-3-138 Sugimoto, Sumiyoshi-ku, \\ Osaka 558-8585, Japan \\ ${ }^{2}$ The OCU Advanced Research Institute for Natural Science and Technology (OCARINA), \\ Osaka City University, Osaka 558-8585, Japan
}

(Received 29 January 2016; accepted 20 June 2016; published online 5 July 2016)

\begin{abstract}
This study provides a method of determining the internal electric field of polymer light emitting diodes (LEDs) in the working condition. The method employs Stark signals induced by triangular shaped pulse biases and enables estimates of the internal field in a broad voltage region. The internal field under forward bias is shown to be determined by the screening effect caused by injected carriers. Spatial distribution calculated for the LED suggests the presence of strong electric field formed by accumulated carriers near the electrodes. The proposed method is applicable to a variety of devices and can promote understanding of veiled roles of internal fields on device operation. Published by AIP Publishing. [http://dx.doi.org/10.1063/1.4955126]
\end{abstract}

Organic semiconductor devices, including light emitting diodes (LEDs), solar cells, and field effect transistors, are still being evolved toward establishing all-organic flexible devices and their applications in a variety of fields. ${ }^{1,2}$ Such organic devices are mostly driven by external bias voltages. Therefore revealing the role of electric field on device operation is at the heart of organic electronics. The internal electric field of the devices is often simply estimated from an external voltage divided by the thickness. However, it could considerably differ from an actual internal field in the bias range where charge injection occurs, because the injected charges may distort the field by space-charge effects ${ }^{3,4}$ and/ or by screening effects. ${ }^{5,6}$ The reason of using such a simply estimated field is precisely because of difficulty in determining the field in the injection range. Several methods have been proposed for estimating the internal field and electric potential. A dc-bridge formed by inserting mediate electrodes inside the device was used to measure the internal potential of organic LEDs (OLEDs). ${ }^{7,8}$ However, the method is possible only for relatively thick devices (e.g., $400 \mathrm{~nm}$ and $800 \mathrm{~nm}$ (Refs. 7 and 8)) and the inserted electrode could affect an internal potential profile. The use of light from "electric field induced second-harmonic generation" (EFISHG) was shown to be effective for measuring the electric field inside OLEDs even in the injection region. ${ }^{9,10}$ However, the technique requires elaborate optical experiments and has now only been realized for specific OLEDs consisting of layered molecule-based materials.

It is well-known that Stark signals appear in electromodulation spectra of organic films proportional to the square of electric field. ${ }^{11}$ The Stark signals can be simply observed by employing lock-in detection techniques combined with bias modulation and were exploited to measure the internal

\footnotetext{
a) Author to whom correspondence should be addressed. Electronic mail: kkane@sci.osaka-cu.ac.jp.

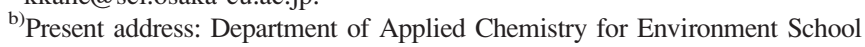
of Science and Technology, Kwansei Gakuin University, 2-1 Gakuen, Sanda, Hyogo 669-1337, Japan.
}

field of organic devices. ${ }^{12,13}$ Since then, the Stark method has been established as a powerful tool to determine the built-in electric field and used for a variety of organic devices such as LEDs and solar cells. ${ }^{5,6,14-26}$ In the method, a linear relation between the internal field and applied biases is generally identified below the built-in potential. However, the Stark signals determining the field were shown to remarkably decrease above a bias where charge injection occurs in polymer LEDs ${ }^{5,6,15,16}$ and light-emitting electrochemical cells. ${ }^{18}$ These observations provide evidence of injected carriers to screen internal fields inside the devices. We note, however, that the lock-in based Stark method cannot determine the magnitude of electric field in the case of field-screening. It is because this method is allowed only for a bias range where the internal field linearly changes for the applied bias, as explained later. Therefore, eventually, the conventional Stark method could not be applicable for devices under working conditions.

In this article, we propose a method of determining the internal field of thin film devices that is available regardless of the presence or absence of the screening effect. The method employs Stark signals induced by applying triangular pulse biases. The intensity of Stark signals is demonstrated to vary according to theoretical predictions, by which we determine the field-bias characteristics of a polymer LED. The characteristics demonstrate clearly the presence of the screening effect, and we finally estimate a spatial distribution of electric field inside the LED. The presence of screening effect can seriously affect interpretations on the operation mechanism of organic devices such as LEDs and solar cells. Systematic studies based on the method proposed in this study would be effective for understanding the underlying device physics.

The structure of the polymer LED used in this study is ITO/PEDOT-PSS/MEH-PPV/Ca/Al, where PEDOT-PSS is $\operatorname{poly}(3,4-e t h y l e n e d i o x y t h i o p h e n e)-p o l y($ styrenesulfonate) and MEH-PPV is poly[2-methoxy,5-(2'-ethyl-hexyloxy)-pphenylene vinylene]. The MEH-PPV layer was spin-coated 
in a glovebox from the chlorobenzene solution at $5 \mathrm{mg} / \mathrm{ml}$. The thickness of the polymer layer was about $50 \mathrm{~nm}$. The Ca and $\mathrm{Al}$ layers at thicknesses of $5 \mathrm{~nm}$ and $20 \mathrm{~nm}$, respectively, were deposited by vacuum evaporation and formed a semitransparent cathode. The active area of the LED was approximately $0.8 \mathrm{~cm}^{2}$. The spectra of bias-modulation experiments were recorded by applying a square-wave AC bias to the LED and detecting the modulation signals with phasesensitive lock-in techniques for the transmitted probe light passing through a monochromator. The probe light was produced using a tungsten/halogen lamp and detected with a Siphotodiode. Transient optical signals were recorded using a digital oscilloscope while applying triangular shape biases $(70 \mathrm{~Hz})$. All measurements were performed at room temperature under vacuum conditions.

The intensity of Stark signals proportional to the normalized transmittance change $(\Delta T / T)$ increases depending on the square of internal electric field $F$ according to the relation

$$
\Delta T / T \propto \operatorname{Im} \chi^{(3)} F^{2},
$$

where $\operatorname{Im} \chi^{(3)}$ is the imaginary component of the third-order susceptibility. In typical experiments to measure the internal field, a bias consisting of DC- $\left(V_{d c}\right)$ and $\mathrm{AC}-\left(V_{a c}(\omega)\right)$ components, $V=V_{d c}+V_{a c}(\omega)$, is applied to devices. ${ }^{13} I_{s}$ is then given by the next relation

$$
\Delta T / T \propto \operatorname{Im} \chi^{(3)}\left\{F_{d c}^{2}+F_{a c}(\omega)^{2}+2 F_{d c} F_{a c}(\omega)\right\} .
$$

$F_{d c}$ and $F_{a c}(\omega)$ are the DC- and AC-components of internal field induced by $V_{d c}$ and $V_{a c}(\omega)$, respectively. $F_{d c}$ includes the contribution from the built-in electric field $F_{b i}$. A lock-in output at $\omega$ in applying a bias $V$ is proportional to the product $F_{d c}\left|F_{a c}(\omega)\right|$ of Eq. (2). The bias-dependence of internal field is obtained from the relation of $F_{d c}$ and $V_{d c}$ measured when sweeping $V_{d c}$ while keeping the $V_{a c}(\omega)$-amplitude constant. This method has been shown to be effective to determine the internal field including $F_{b i}$ of organic devices., ${ }^{5,6,12-26}$ However, in fact, this method includes two issues. First, since $F_{d c}$ is obtained from the lock-in output of $F_{d c}\left|F_{a c}(\omega)\right|$ averaged for the modulation of $V_{a c}(\omega)$, the $V_{a c}$-amplitude should be ideally negligibly small to define the field at $V_{d c}$. Modulation amplitudes often used were $0.5-1 \mathrm{~V}$, which could result in serious inaccuracy. The other issue also arises from use of modulation. In using Eq. (2), $\left|F_{a c}(\omega)\right|$ is assumed to be constant during sweeping $V_{d c}$ under the constant $V_{a c}(\omega)$ modulation. This assumption is valid when the resultant internal field changes linearly during the $\pm V_{a c}$ modulation, typically in the case of the internal field simply consisting of the sum of built-in and external fields. However, the assumption of the constant $-\left|F_{a c}(\omega)\right|$ is not allowed for the internal field changing non-linearly during the $\pm V_{a c}$-modulation because the non-linearity results in changing $\left|F_{a c}(\omega)\right|$ during sweeping $V_{d c}$. Therefore, the lock-in method of field determination cannot be used for bias regions where field screening by charge injection occurs, suggesting inapplicability under operating conditions of LEDs.

From the reasons above, we propose a determination method of internal field without using modulation techniques, which is available even in the bias range of charge injection. In case of no modulation, the Stark intensity simply changes depending on the square of internal field according to Eq. (1). We hereafter show that such Stark signals can be picked up directly from bias-dependent spectral signals by careful spectral analyses and experiments using triangular shaped voltage biases.

A bias to devices in the injection region can contribute both to applying an electric field to molecules inside devices and generating excited species such as carriers and excitons. The MEH-PPV diode indeed exhibits spectral features from both effects, as reported previously. ${ }^{18}$ Resolving the coexistent spectral features is essential for precisely examining spectral features of Stark signals, including cases of using modulation techniques although such coexistent structure has not been well-studied. Generally, coexistent components of different origins may be decomposed by regulating a modulation frequency and lock-in phase, because they often have different response rates to the bias modulation. ${ }^{15,27}$ Indeed as shown in Fig. 1, by regulating a lock-in phase of the modulation signals obtained under a relatively high frequency (12 $\mathrm{kHz}$ ), a particular spectral component was resolved in the quadrature phase. The quadrature spectrum is similar to the differential absorption spectrum of MEH-PPV film and also similar to the Stark spectra of MEH-PPV obtained from electroabsorption (EA) measurements with coplanar electrodes ${ }^{28}$ and those reported previously. ${ }^{11,29,30}$ The resolved quadrature spectrum thus corresponds to the Stark spectrum of the present MEH-PPV diode.

The spectral structure below $2.0 \mathrm{eV}$ of the in-phase signal in Fig. 1 does not appear in the Stark spectrum of the quadrature phase and is similar to that of polarons generated by the bias application. ${ }^{31}$ The signals below $2.0 \mathrm{eV}$ thus mainly originate from charged species. The in-phase spectrum consists

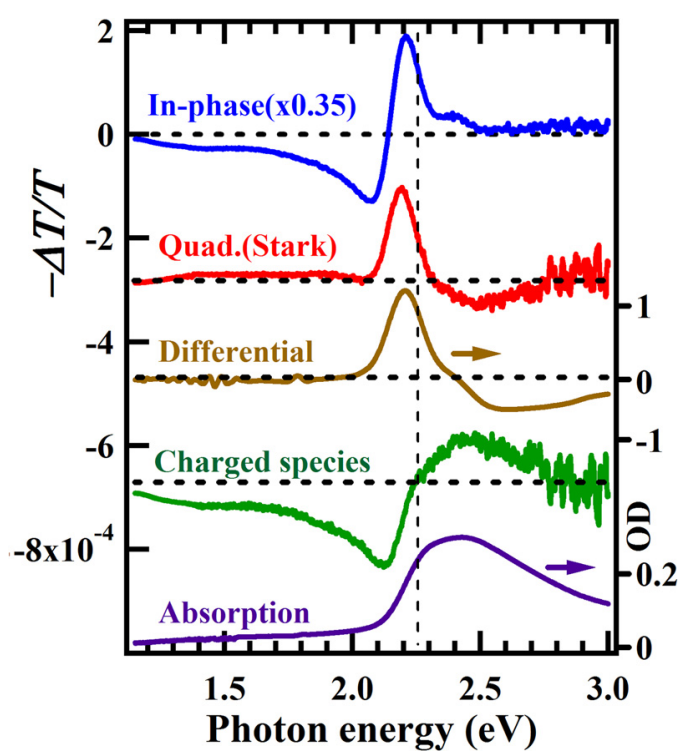

FIG. 1. Optical spectra obtained by applying square-wave bias of $4 \mathrm{~V}$ to the polymer LED at $12 \mathrm{kHz}$. From the top, in-phase and quadrature spectra measured by lock-in techniques, the differential steady-state absorption spectrum, the spectrum of charged species obtained by subtracting the Stark signal from the in-phase spectrum, and the steady-state absorption spectrum. The vertical broken line indicates the energy position $(2.27 \mathrm{eV})$ where the signal from the charged species is negligible. 
of the sum of the signals from charged species and Stark component, suggesting that the spectrum of charged species can be decomposed by subtracting the Stark component. Figure 1 presents the spectrum of charged species obtained by moderately subtracting the Stark components from the in-phase spectrum (see supplementary material ${ }^{28}$ ). The spectrum has an upward peak at $2.45 \mathrm{eV}$ and downward peak at $2.15 \mathrm{eV}$ attributed to bleaching and absorption signals, respectively. The bleaching component is similar to the absorption spectrum of MEH-PPV, indicating relevance of the spectral decomposition. Comparing the spectra of Stark signals and the charged species, it turns out that the signal intensity around $2.27 \mathrm{eV}$ of the charged species is negligibly small but that of the Stark signals is detectable. Therefore, we can expect that bias-induced signals measured at this energy enable independent detection of the Stark signals.

The bias-dependent feature of the Stark signals was thus examined at $2.27 \mathrm{eV}$. We then employed a triangular shape bias shown in Fig. 2(a) for quasi-synchro-measurements of Stark intensity in a wide bias range. The intensity of Stark signals is typically an order of $10^{-5}$ to $10^{-3}$ of transmitted light and the signal-detection is usually difficult without lock-in techniques because of the fluctuation of background signals. The measurements by the triangular bias were thus repeatedly made to average slowly fluctuating background signals. Figure 2(a) indicates the time-traces of spectroscopic signals measured at $2.27 \mathrm{eV}$ and current induced by linearly varying biases from -3 to $10 \mathrm{~V}$. Upon increasing the voltage from $-3 \mathrm{~V}$, the current rises around $3 \mathrm{~ms}$ and the spectroscopic signals then exhibit a peak. Such time traces can be directly converted into the characteristics of bias-current and bias-spectroscopic signals, and their characteristics in the bias-increasing process are shown in Fig. 2(b).

The time traces of spectroscopic signals were also measured at other energy points and showed that the time trace at $2.27 \mathrm{eV}$ results from Stark signals, ${ }^{28}$ consistent with the spectral analyses in Fig. 1. The signal intensity at $2.27 \mathrm{eV}$ is thus predicted to exhibit squared bias-dependence according to Eq. (1) in the bias region of negligible charge injection. Also the signal is expected to have a maximum or minimum peak at the built-in potential $\left(V_{b i}\right)$ because the Stark signal is then nulled. The characteristics at $2.27 \mathrm{eV}$ in Fig. 2(b) exhibit a peak at $2.2 \mathrm{~V}$, around which the current starts to rise. The peak bias fairly agrees with $V_{b i}$ calculated from the work function difference of $\mathrm{Ca}(-2.8 \mathrm{eV})$ and PEDOT-PSS $(-4.8-5.0 \mathrm{eV})$. Moreover, the squared bias-dependence of spectral signals was also examined based on the center voltage assumed to be $2.2 \mathrm{~V}$ and the result fitted below $2.2 \mathrm{~V}$ is shown in Fig. 2(b). It clearly demonstrates the proportionality of the spectral intensity to the squared voltage. Therefore, both results of the peak position and bias-dependence are consistent with the theoretical prediction. This confirms that the characteristics at $2.27 \mathrm{eV}$ enable determination of internal field.

Unlike the case of using bias-modulation techniques, the characteristics shown above are applicable to the fielddetermination regardless of the presence or absence of charge injection. For the determination, the bias-dependent part of the characteristics at $2.27 \mathrm{eV}$ was extracted and its square root was calculated by setting the peak point at $2.2 \mathrm{~V}$ as the origin. The result was directly converted into the internal field using the identified squared bias-dependence below $2.2 \mathrm{~V}$ and is shown in Fig. 3. It determines the magnitude of internal field including the bias region of carrier injection. As shown in the bias-dependence, the internal field immediately changes above $V_{b i}$ and becomes saturated. This saturation is definite evidence of the field-screening occurring by injected carriers. The field under the screening bias is determined to be $0.2 \mathrm{MV} / \mathrm{cm}$. We here note that, if the lock-in method of field determination is applied for the screening region indicated in Fig. 3, the screening field could be misregarded as zero because the lock-in signals in the quasi-constant field region are nulled. We also note that the internal field could

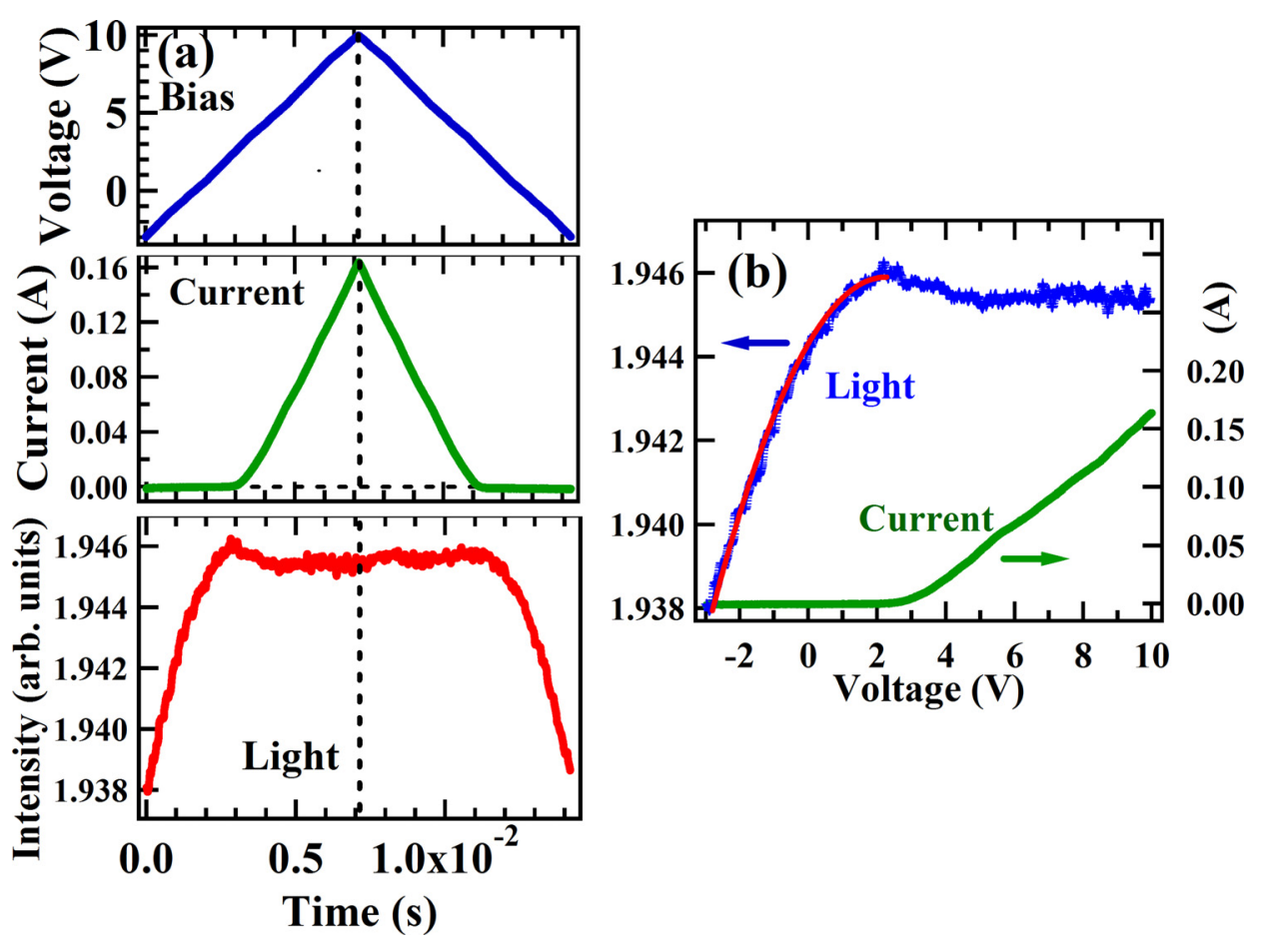

FIG. 2. (a) Time traces of the triangular shape bias, the bias-induced current, and the bias-induced light intensity measured at $2.27 \mathrm{eV}$. (b) Characteristics of bias-current and bias-light intensity obtained from the time trace of (a). The red curve for the light intensity indicates the fit indicating squared bias dependence of the intensity. 


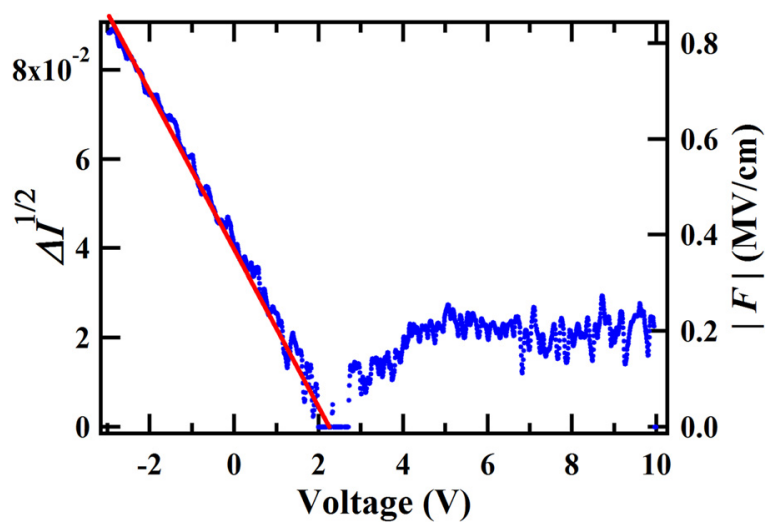

FIG. 3. Bias-dependence of the square root for the light intensity $\left(\Delta I^{1 / 2}\right.$ : left axis) and the calculated magnitude of internal electric field (right axis). The red solid line is the linear fit.

not be uniform inside the diode typically due to space-charge effects by the injected carriers. Such non-uniform fields could cause spatially dependent spectral responses of EA signals, as previously shown for inorganic semiconductors. ${ }^{32}$ The non-uniformity can also distort the lineshape of EAspectrum due to mixing of complex dielectric components. ${ }^{32}$ However, in the present case, the Stark-component spectrum obtained under forward bias resembled the EA-spectrum, suggesting negligible distortion of lineshape. Therefore, the field non-uniformity in this system would be relatively small. The determined field then indicates the root-mean square (RMS) of spatially distributed fields according to Eq. (1). Since an RMS value is generally larger than a mean value, the mean saturation field could be somewhat smaller than $0.2 \mathrm{MV} / \mathrm{cm}$.

The screening field is oppositely directed to the external field and should thus be formed by distributions of holes and electrons increasing toward the counter electrodes. Such carrier distributions are not expected in conventional conduction mechanisms like a space charge limited conduction that assumes reduction of carrier density with increasing distance from injection electrodes. Therefore, the screening should be due to accumulation of injected carriers at the counter electrodes following carrier transport. Brewer et al. also proposed such carrier accumulation for explaining the field screening suggested from their experiments for polyfluorene (PFO)-based LEDs and concluded that the accumulation of electrons at the anode would be the main cause of the screening. ${ }^{5}$ Similar electron-accumulation can also occur in MEH-PPV LEDs. However, since the mobility of electrons in MEH-PPV is much smaller than that of holes, ${ }^{33}$ many electros may not reach the anode in low biases near $5 \mathrm{~V}$ where the field screening occurs. Therefore, we regard hole-accumulation near the cathode as being the major origin of the screening.

Since the potential drop in the PEDOT-PSS layer is negligible, the determined saturation field $(0.2 \mathrm{MV} / \mathrm{cm})$ only corresponds to a potential drop of $1 \mathrm{~V}$ for the 50 -nm thickness, which is much smaller than the bias for screening $(>5 \mathrm{~V})$. The rest of potential drop is expected to occur at the accumulation layers of holes and/or electrons. We here neglect for simplicity field-distributions by possible space charge effects and assume that the accumulation layers are so thin as not to yield the Stark signals. Spatial distributions of the internal field and electric potential can then be estimated as a function of distance $(x)$ from the anode by using the Gauss's law for the accumulation layers: $\mathrm{d} F / \mathrm{d} x=\rho / \varepsilon$, with $\varepsilon$ and $\rho$ being the permittivity and the charge density of accumulated carriers neglecting the density of mobile carriers, respectively. Figures 4(a) and 4(b) show the calculated distributions of potential and internal field under $5 \mathrm{~V}$ and $10 \mathrm{~V}$-biases assuming only hole-accumulation with a uniform carrier distribution in the accumulation layer. ${ }^{28}$ The potential distribution indicates rapid potential drop near the cathode following the gradual $1 \mathrm{~V}$-reduction. The rapid potential drop then causes a rapid increase of electric field. We here note that the observed field-saturation is probably maintained by increasing accumulated carriers during increasing the bias, which also results in increasing the thickness of accumulation layer. The increased thickness by the bias enhances the internal field within the accumulation layer, as shown by the difference of field-magnitude between $5 \mathrm{~V}$ - and $10 \mathrm{~V}$-biases in Fig. 4(b). We emphasize that the increased internal field could induce emission of accumulated carriers into the electrodes, resulting in partial participation of the accumulated carriers in the electric conduction. Therefore, careful considerations of the role of accumulated carriers would be required to consider the conduction mechanism.

The hole-accumulation near the cathode could be due to electric fields formed by Schottky contact of polymers with the Ca-cathode that are directed in opposition to external fields. Similar fields could also be formed at the anode for electron-accumulation. Therefore, the degree of accumulation, or of screening, would depend on combination of materials used for active layers and electrodes. We finally emphasize that the determination method in this study can be much simply applied than the present case depending on the degree of the spectral overlap between Stark signals and signals from bias-induced species.

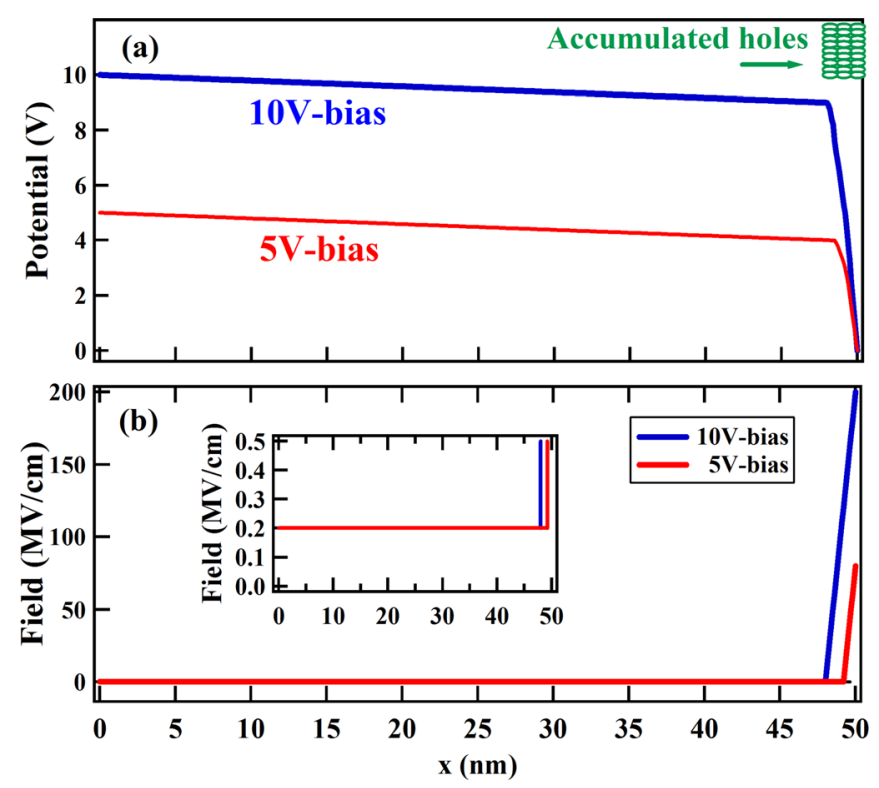

FIG. 4. Spatial distributions of the potential (a) and internal field (b) calculated for the case of $5 \mathrm{~V}$ - and $10 \mathrm{~V}$-biases assuming a uniform distribution of the accumulated holes. The inset in (b) is an enlarged view of the field for indicating the small field value. 
In summary, this study proposed the method of determining the internal field of polymer LEDs from spectroscopic measurements. The bias-characteristics of spectroscopic signals were demonstrated to change according to theoretical predictions, confirming the relevance of the method. The internal field determined was shown to become saturated because of screening effects. The spatial distributions of the field and potential predict that the electric field near the electrodes increases with increasing the bias and affects the electric conduction.

This work was supported in part by JSPS KAKENHI Grant Nos. 25288117 and 26620207.

${ }^{1}$ S. Bauer, S. Bauer-Gogonea, I. Graz, M. Kaltenbrunner, C. Keplinger, and R. Schwoediauer, Adv. Mater. 26, 149 (2014).

${ }^{2}$ Handbook of Flexible Organic Electronics, edited by S. Logothetidis (Elsevier, Cambridge, 2014).

${ }^{3}$ M. A. Lampert and P. Mark, Current Injection in Solids (Academic Press, New York, 1970).

${ }^{4}$ H. Li, L. Duan, D. Zhang, and Y. Qiu, J. Phys. Chem. C 118, 9990 (2014).

${ }^{5}$ P. J. Brewer, P. A. Lane, A. J. deMello, D. D. C. Bradley, and J. C. deMello, Adv. Funct. Mater. 14, 562 (2004).

${ }^{6}$ P. A. Lane, P. J. Brewer, J. Huang, D. D. C. Bradley, and J. C. deMello, Phys. Rev. B 74, 125320 (2006).

${ }^{7}$ M. Hiramoto, K. Koyama, K. Nakayama, and M. Yokoyama, Appl. Phys. Lett. 76, 1336 (2000).

${ }^{8}$ X. R. Yin, Y. K. Le, X. D. Gao, Z. Y. Sun, and X. Y. Hou, Appl. Phys. Lett. 97, 153305 (2010).

${ }^{9}$ T. Manaka, E. Lim, R. Tamura, and M. Iwamoto, Nat. Photonics 1, 581 (2007).

${ }^{10}$ M. Weis, T. Otsuka, D. Taguchi, T. Manaka, and M. Iwamoto, J. Appl. Phys. 117, 155503 (2015).

${ }^{11}$ M. Liess, S. Jeglinski, Z. V. Vardeny, M. Ozaki, K. Yoshino, Y. Ding, and T. Barton, Phys. Rev. B 56, 15712 (1997).

${ }^{12}$ I. H. Campbell, M. D. Joswick, and I. D. Parker, Appl. Phys. Lett. 67, 3171 (1995).
${ }^{13}$ I. H. Campbell, T. W. Hagler, D. L. Smith, and J. P. Ferraris, Phys. Rev. Lett. 76, 1900 (1996).

${ }^{14}$ J. C. deMello, J. J. M. Halls, S. C. Graham, N. Tessler, and R. H. Friend, Phys. Rev. Lett. 85, 421 (2000).

${ }^{15}$ P. A. Lane, J. C. deMello, R. B. Fletcher, and M. Bernius, Appl. Phys. Lett. 83, 3611 (2003).

${ }^{16}$ P. J. Brewer, A. J. deMello, J. C. deMello, P. A. Lane, D. D. C. Bradley, R. Fletcher, and J. O'Brien, J. Appl. Phys. 99, 114502 (2006).

${ }^{17}$ V. Bodrozic, T. M. Brown, S. Mian, D. Caruana, M. Roberts, N. Phillips, and J. J. Halls, Adv. Mater. 20, 2410 (2008).

${ }^{18}$ C. V. Hoven, J. Peet, A. Mikhailovsky, and T.-Q. Nguyen, Appl. Phys. Lett. 94, 033301 (2009).

${ }^{19}$ M. Zhou, L.-L. Chua, R.-Q. Png, C.-K. Yong, S. Sivaramakrishnan, P.-J. Chia, A. T. S. Wee, R. H. Friend, and P. K. H. Ho, Phys. Rev. Lett. 103, 036601 (2009).

${ }^{20}$ R. J. de Vries, S. L. M. van Mensfoort, R. A. J. Janssen, and R. Coehoorn, Phys. Rev. B 81, 125203 (2010).

${ }^{21}$ F. So and D. Kondakov, Adv. Mater. 22, 3762 (2010).

${ }^{22}$ K. Kanemoto, A. Ogata, N. Inoue, T. Kusumoto, H. Hashimoto, I. Akai, and T. Karasawa, Appl. Phys. Lett. 97, 033307 (2010).

${ }^{23}$ B. A. MacLeod, N. E. Horwitz, E. L. Ratcliff, J. L. Jenkins, N. R. Armstrong, A. J. Giordano, P. J. Hotchkiss, S. R. Marder, C. T. Campbell, and D. S. Ginger, J. Phys. Chem. Lett. 3, 1202 (2012).

${ }^{24}$ O. Pachoumi, C. Li, Y. Vaynzof, K. K. Banger, and H. Sirringhaus, Adv. Energy Mater. 3, 1428 (2013).

${ }^{25}$ B. Liu, R.-Q. Png, J.-K. Tan, and P. K. H. Ho, Adv. Energy Mater. 4, 1200972 (2014).

${ }^{26}$ E. Siebert-Henze, V. G. Lyssenko, J. Fischer, M. Tietze, R. Brueckner, T. Menke, K. Leo, and M. Riede, Org. Electron. 15, 563 (2014).

${ }^{27}$ K. Kanemoto, M. Yasui, D. Kosumi, A. Ogata, M. Sugisaki, T. Karasawa, I. Akai, and H. Hashimoto, Phys. Rev. Lett. 103, 187402 (2009).

${ }^{28}$ See supplementary material at http://dx.doi.org/10.1063/1.4955126 for additional results and detailed descriptions of some experiments.

${ }^{29}$ S. J. Martin, D. D. C. Bradley, P. A. Lane, H. Mellor, and P. L. Burn, Phys. Rev. B 59, 15133 (1999).

${ }^{30}$ A. K. Tripathi and Y. N. Mohapatra, Phys. Rev. B 84, 205213 (2011).

${ }^{31}$ T. Takahashi, K. Kanemoto, M. Kanenobu, Y. Okawauchi, and $\mathrm{H}$. Hashimoto, Sci. Rep. 5, 15533 (2015).

${ }^{32}$ D. E. Aspnes and A. Frova, Solid State Commun. 7, 155 (1969).

${ }^{33}$ Y. Zhang, B. de Boer, and P. W. M. Blom, Phys. Rev. B 81, 085201 (2010). 